\title{
The Application of Digital Image Processing Method in Range Finding by Camera
}

\author{
Chunyan $\mathrm{Xie}^{1,{ }^{*}}$, Haibin $\mathrm{Ma}^{2}$ \\ ${ }^{1}$ College of Information Technology, Hebei Normal University, Shi Jiazhuang, China \\ ${ }^{2}$ College of Vocational and Technology, Hebei Normal University, Shi Jiazhuang, China
}

\begin{abstract}
For the disadvantage of contact method in range finding, such as physical effects, high requirements of environment, operating errors and so on, a different method is proposed as required--digital image processing for range finding. This non-contact method could be finished after the collection and handling of laser spots by USB camera, the extraction of feature information of image, and the building of the Quantitative relationship between feature and measured displacement. It is available and practical by experiment without the complex marking procession of camera system.
\end{abstract}

Keywords: Camera, Image processing, Non-contact measurement, Ranging.

\section{INTRODUCTION}

In the process of monitoring the flight attitude of the aircraft, the space location is one of the basic information, and can be obtained by sensor. It is a kind of range finding in space location, which has so many methods to complete. In the field of aerospace, because of the limitation of their factors, such as volume, quality, installation conditions and structure, the traditional contact sensors are gradually replaced by non-contact measure. With the advantages, such as higher speed, richer information, and less impact on the object, the method based on digital image processing attracts more attention. This paper presented a non-contact measurement for range finding in the research of the space location of adaptive wing. By using the images of the laser spots captured by digital cameras as an information carrier, using the processing method, such as image preprocessing, threshold segmentation and feature extraction, and other related digital image processing methods, the quantitative relation can be got between the displacement information and the extracted image features by using least-squares curve fitting [1-3].

\section{DIGITAL IMAGE PROCESSING}

In the process of measurement, with the changes on laser's projection distance, the spot by laser reflection would drift due to temperature changes, environmental vibration, and air turbulence, and finally be irregular with the impact of scattering, diffraction, other interference light (natural light), and the irregular reflection characteristics because of roughness on the surface. Thereby the accuracy of collection and operation is not so good. For this reason, the essay used the digital image processing method as shown in Fig. (1).

\footnotetext{
*Address correspondence to this author at the College of Information Technology, Hebei Normal University, Shi Jiazhuang, China; Tel: 13832067230; E-mail: xiecynu@163.com
}

In the process of image generation, transmission, sampling and quantization make the quality of image decreased, due to the impact of light, random noise and others factors on the stability of the imaging system. While the image preprocessing could improve the quality by enhancing useful information, or reducing noise interference.

Because the multiplicative nonlinear noise is the primary problem, median filtering method is the best way to achieve image preprocessing [4-6]. Turky pointed that median filtering could reduce noise and remains the image details and sharp edges at the same time. The basic principle is that the value in the digital image is expressed in the median value of points in the neighborhood field. The median is defined as follows:

the numbers $\mathrm{x} 1, \mathrm{x} 2, \mathrm{x} 3, \ldots \mathrm{xn}$, and $\mathrm{xi} 1<=\mathrm{xi} 2<=\mathrm{xi} 3<=\ldots<=\mathrm{xin}$, then $\mathrm{y}=\operatorname{Median}(\mathrm{x} 1, \mathrm{x} 2, \mathrm{x} 3, \ldots, \mathrm{xn})$

$\mathrm{y}$ is the median value of the sequence of $\mathrm{x} 1, \mathrm{x} 2, \mathrm{x} 3, \ldots, \mathrm{xn}$.

As for two-dimensional digital images, $\{x i j,(I, j) \in I 2\}$ means digital gray value of each point in images. The twodimensional median filter can be defined as (Filtering window is A):

$Y i j=\operatorname{Median}\left\{\mathrm{x}^{\mathrm{ij}}\right\}=\operatorname{Median}\left\{\mathrm{x}^{(\mathrm{i}+\mathrm{r}),(\mathrm{j}+\mathrm{s})},(\mathrm{r}, \mathrm{s}) \in \mathrm{A},(\mathrm{I}, \mathrm{j}) \in \mathrm{I}^{2}\right\}$

Two-dimensional Median filtering window can take a square, circular or cross shape. Algorithm flow is shown in Fig. (2).

Image segmentation is one important processing step before image analysis. In order to describe the image and extract useful information, it divides the image space into several regions. In the same region, the characteristics of the image are similar, and in different regions, the characteristics of the image go far away. The methods of segmentation used commonly are region-based segmentation, boundary-based segmentation and the segmentation based on a combination of regional and border. The choice between the methods of 


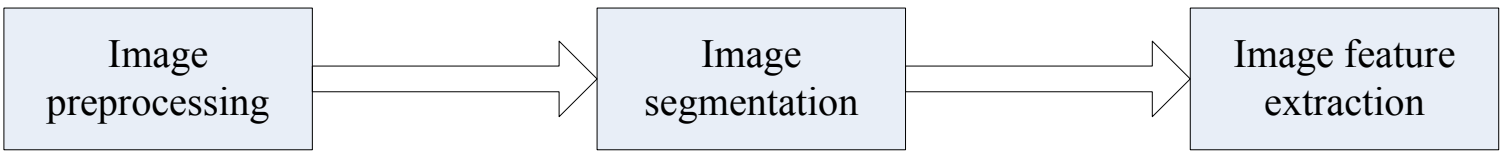

Fig. (1). The flowchart of digital image processing

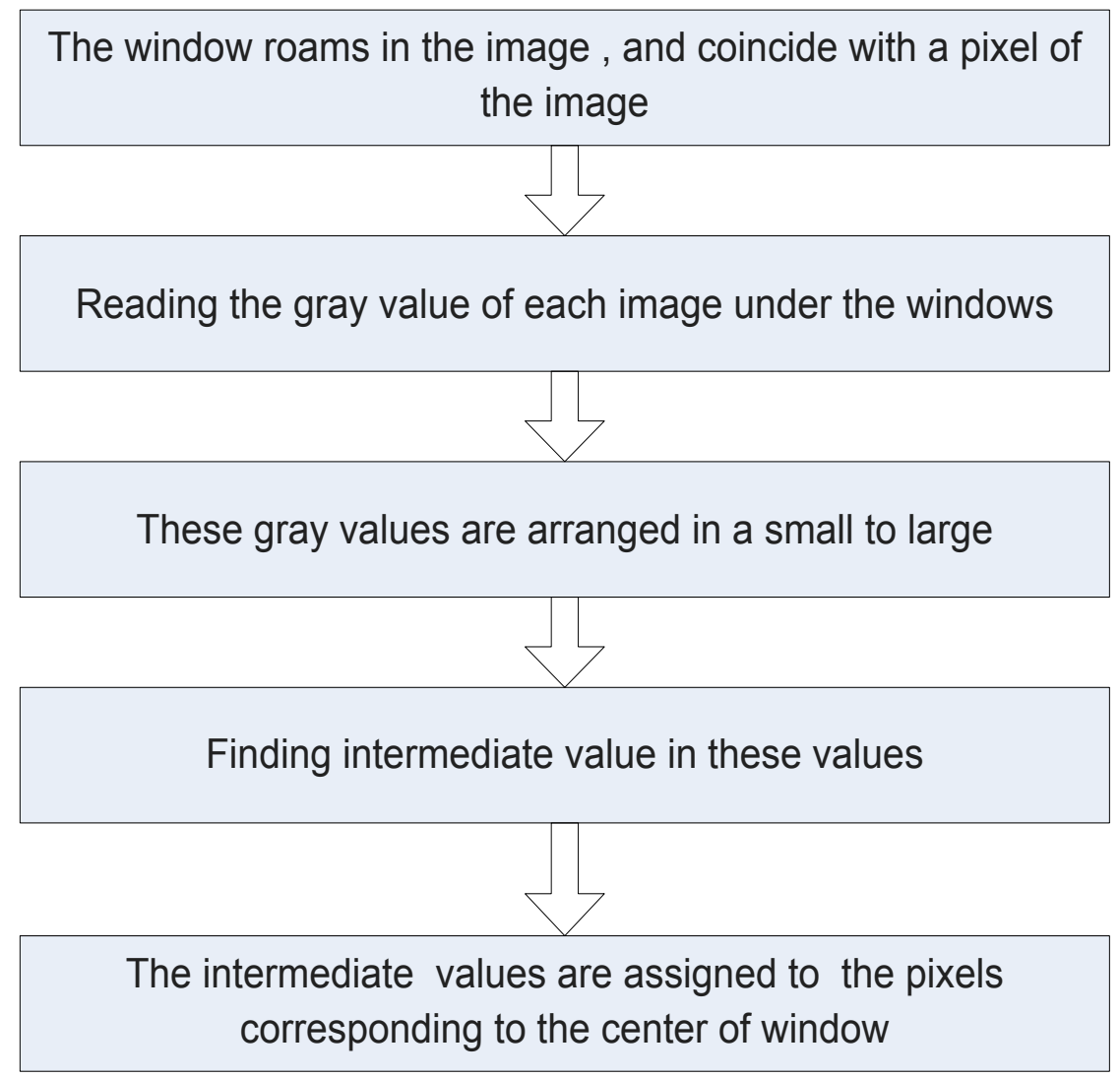

Fig. (2). The flowchart of intermediate value filtering algorithm.

segmentation has no criteria, and so the proper method should be selected according to the specific object in real application. Because the target and the background in the laser reflection image lie in the different gray level, the threshold segmentation method based on gradation histogram curve fitting is put forward, which is based on the region-based segmentation $[7,8]$.

Threshold segmentation is a method of image segmentation, and plays an important role in the application of image segmentation because of its visuality and simplicity. It not only compresses the large amount of data, but also simplifies the analysis and processing steps greatly. The basic principle is that dividing the image gray into different levels, dividing the set of pixels according to the gray threshold value, and determining the region corresponding to the realistic scene.

The original image $\mathrm{f}(\mathrm{x}, \mathrm{y})$ corresponding to the histogram is shown in Fig. (3). Since the gray level and the background are the main modes, therefore, these two modes are separated by a threshold value $\mathrm{T}$ in order to make the object extracted from the background. The image $\mathrm{g}$ $(\mathrm{x}, \mathrm{y})$ processed by threshold is defined as: $g(x, y)= \begin{cases}0 & f(x, y)<T \\ 1 & f(x, y)>=T\end{cases}$

Among them, the pixel marked 1 is corresponding to the object, and the pixel marked 0 is corresponding to the background.

The key of the threshold segmentation is the choice of gray threshold value $\mathrm{T}$, and because of the method of choosing, many methods could be used, such as Global threshold method, local threshold method, dynamic threshold method and so on [9-11].

After the pre-processing, it could be determined the gradation histogram of image's pixel for the analysis of its features. In the histogram, marking the gray values of target and background is to estimate the preliminary position of threshold peak, and intercepting the corresponding position is to curve fitting. After that, the mineralization of the curve fitting could be got, which is the threshold of the image segmentation, and finally the image binarization could be got 


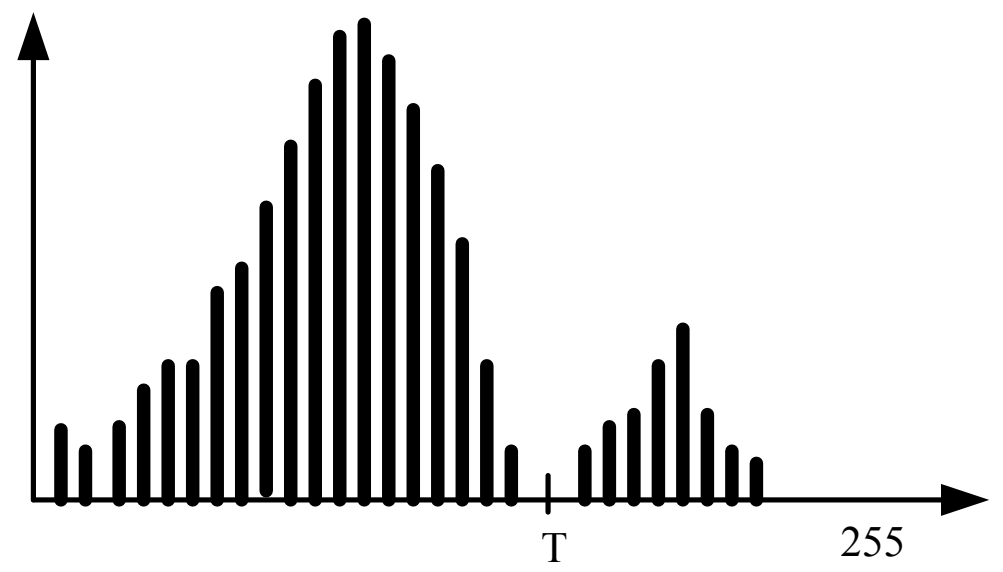

Fig. (3). Gray image histogram.

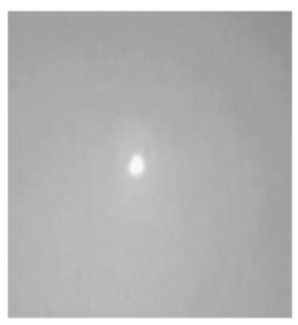

a. original image of laser spo

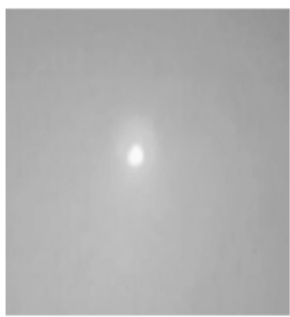

\section{b. The intermediate value gray image filtered}

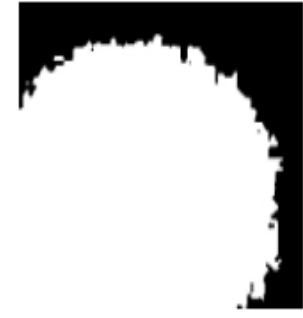

\section{c. The Classic Otsu threshold} segmentation method

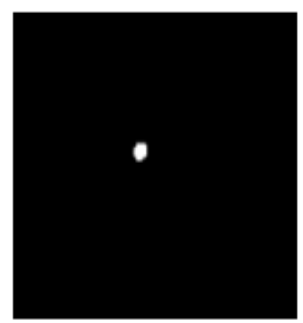

d. The curve fitting threshold segmentation method based on gray histogram

Fig. (4). The results compared with threshold segmentation.

according to this threshold to obtain the binary image and show the characteristics. Referring to the researched laser reflection image, two methods could be used to obtain the threshold segmentation, Otsu classic method and method of curve fitting based on gradation histogram. As the Fig. (4). shows, the method presented in this paper adapts to the segmentation of laser spots better.

Image characteristics mean the outstanding characteristics or basic feature. Feature extraction means extracting the physical features, geometric features and other features from the object, and obtaining the relative parameter. In the procession of measuring, the main attention is paid on obtaining the geometric size of laser spots of the laser reflection, and so it is important to describe and analyze the features of the region from binary image segmentation, such as Area, centroid, Euler number and so on. And finally the characteristics of laser spots could be obtained. The motion information could be obtained after the analysis of the features.

\section{EXPERIMENTAL VERIFICATION}

Principle of the measurement system is shown in Fig. (5). The system consisted of laser transmitter, USB digital camera, target object, and computer. First, the laser transmitter 


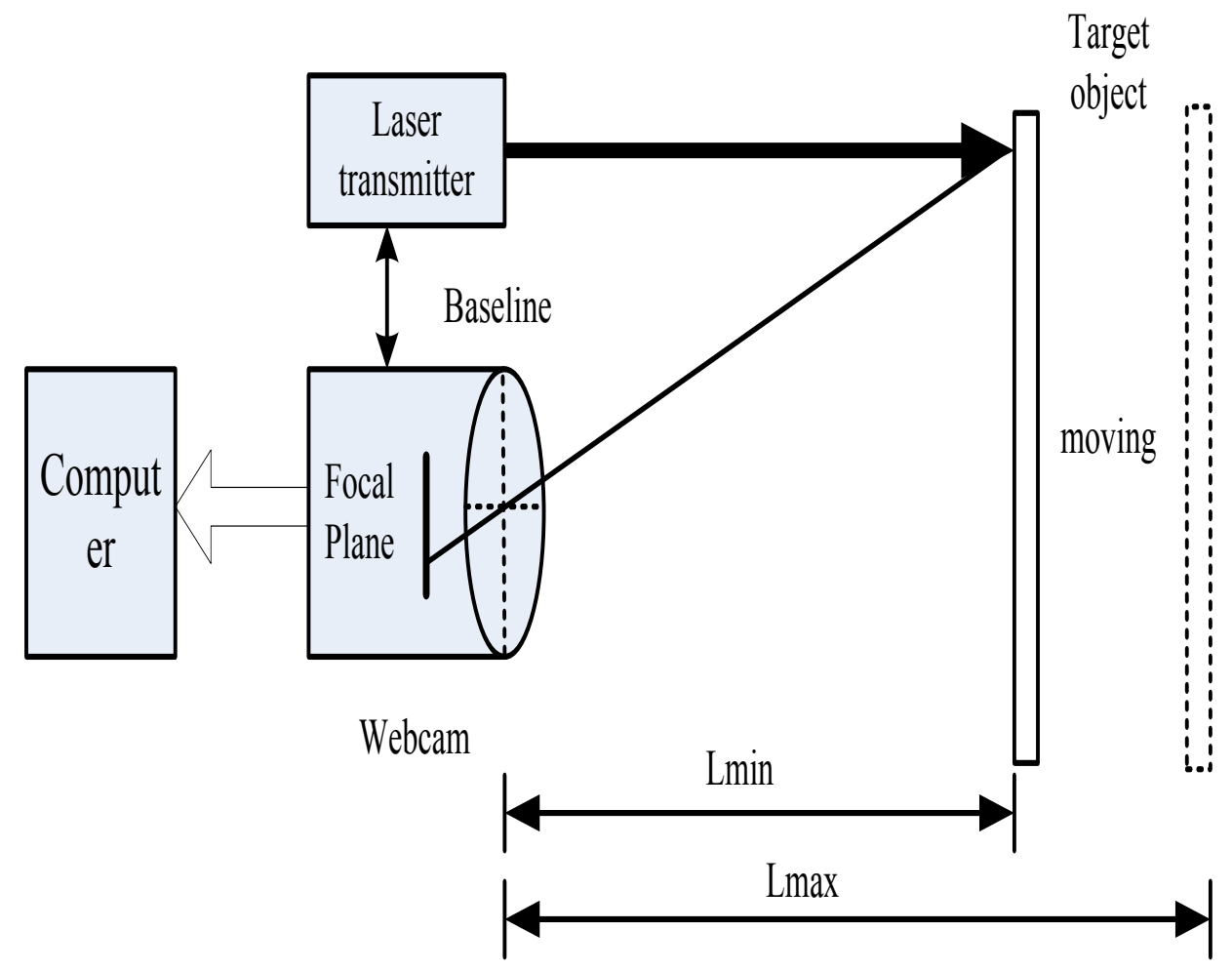

Fig. (5). The schematic diagram of measurement system.

emitted laser beam on the moving object to form the spots after the reflection on the surface of the object. Next USB camera captured the spots on the surface of the object and it should be assured that the camera could capture all images. Finally, the camera sent this image of the spots to the computer.

After the laser transmitter and camera were located and the length of baseline was given, the size of laser spots, that is Pixels, would change with the motion of the target object on the horizontal direction. In the realistic operation, there was a divergence angle of the laser, and so when the target was farther, the laser spots would have a larger diameter. On this principle, The distance between object and the camera could be calculated according to the size of spots by the digital image processing algorithm. Because of the limitation of the size of image by camera, the focal length and laser's characteristics, the range of measurement system should be limited from min distance A to max distance B to make sure the accuracy of the measurement.

The measurement system marked by the realistic physical distance, and the corresponding equivalent relationship could be obtained between marked distance and the size of spots collected in the marked location. Building a curve between the measured distance and the size of spot according to the least-squares curve fitting and then the measurement of displacement could be realized

The process of experiment is as follows: First marking the realistic physical distance between the laser transmitter and object, and collecting the laser reflection image. Second handling the collected image and then transmitting the color image into gray one. And after smoothing by median filter and the threshold segmentation, the gray image would be transformed to binary image, as shown in Fig. (6).

Finally, extracting the area of spots according to the feature description method, and building the curve to show the relation between the distance and size of spot. After finishing all the steps, use the method of least-squares curve fitting to calculate, as shown in Fig. (7).

For the camera in the experiment, CMOS sensors with 300000 pixel were used, and Optical resolution was $640 \times 480$. Imaging distance was more than $5 \mathrm{~cm}$, and it could focus automatically with minimum focus range $15 \mathrm{~cm}$. The maximum number of frames was 30/s. The Quantitative relationship between the distance D and size of laser spots L calculated by curve fitting is as follows:

The measurement distance is $15 \mathrm{~cm} \leq \mathrm{D} \leq 1000 \mathrm{~cm}$.

Note: because of the different hardware equipment and environment, the Quantitative relationship and measurement distance were just available in this experiment.

According to the principle of experiment, three positions should be collected out of the marking range of distance to obtain Reflected laser image. Then according to the corresponding digital image processing algorithm, feature area could be calculated. Thus displacement could be obtained by curve fitting and the error analysis could be done according to actual physical displacement. The results are shown in Tables $\mathbf{1}$ and $\mathbf{2}$. 


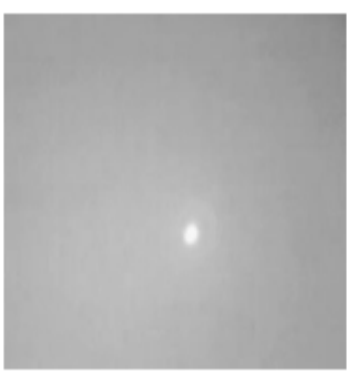

a. original image of laser spot

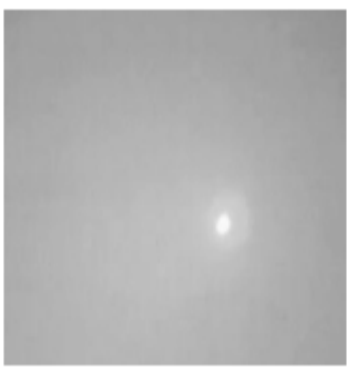

b. The gray image

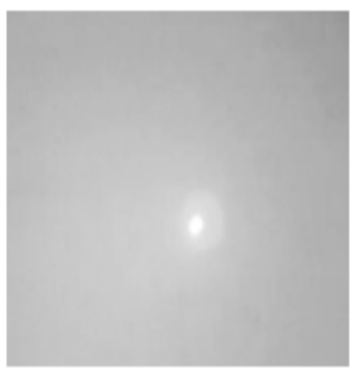

c. intermediate value gray image filtered

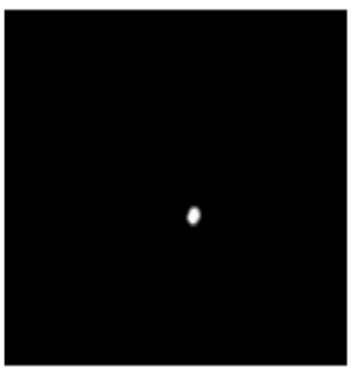

d. The binary image after threshold segmentation

Fig. (6). The images processed by the laser spot.

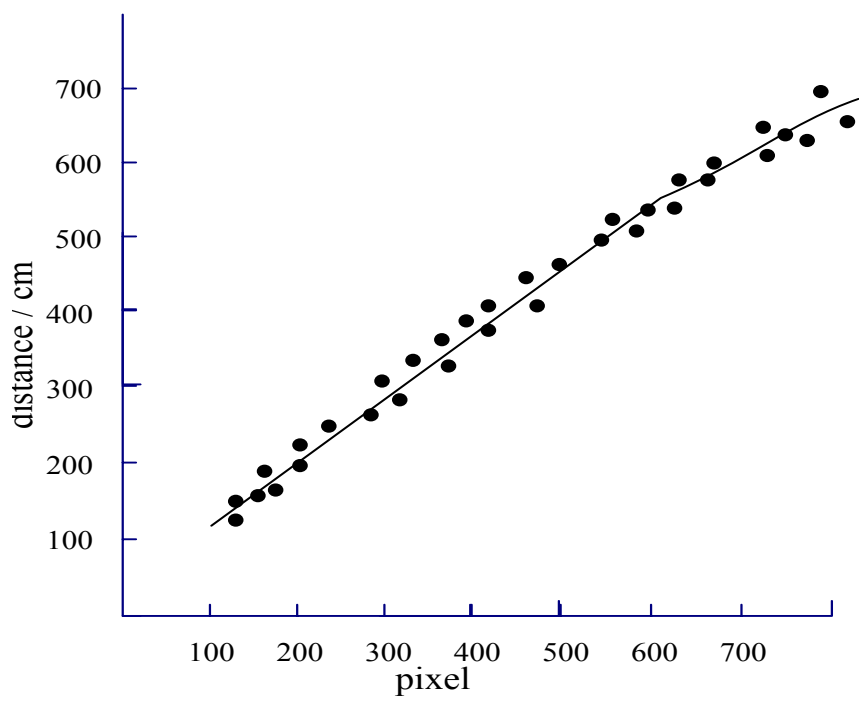

Fig. (7). The least-squares curve fitting.

Table 1. The analysis table of measurement results.

\begin{tabular}{|c|c|c|c|c|}
\hline Experiment & Spot Size (pixel) & Estimated Distance(cm) & Actual Distance(cm) & Relative Error (\%) \\
\hline \hline 1 & 129.875 & 132.205 & 133 & 0.598 \\
\hline 2 & 180.106 & 170.856 & 172 & 0.665 \\
\hline
\end{tabular}


Table 1. contd...

\begin{tabular}{|c|c|c|c|c|}
\hline Experiment & Spot Size (pixel) & Estimated Distance(cm) & Actual Distance(cm) & Relative Error (\%) \\
\hline 3 & 198.205 & 220.023 & 222 & 0.891 \\
\hline 4 & 226.053 & 248.203 & 250 & 0.719 \\
\hline 5 & 298.065 & 318.316 & 320 & 0.526 \\
\hline 6 & 400.023 & 326.008 & 328 & 0.607 \\
\hline 7 & 429.203 & 378.108 & 381 & 0.759 \\
\hline 8 & 490.102 & 410.862 & 413 & 0.518 \\
\hline 9 & 498.003 & 460.716 & 464 & 0.708 \\
\hline 10 & 557.026 & 507.425 & 509 & 0.309 \\
\hline 11 & 579.125 & 516.431 & 519 & 0.495 \\
\hline 12 & 618.864 & 523.801 & 526 & 0.418 \\
\hline 13 & 679.822 & 590.106 & 593 & 0.488 \\
\hline 14 & 709.283 & 610.209 & 614 & 0.617 \\
\hline 15 & 777.419 & 620.116 & 624 & 0.622 \\
\hline
\end{tabular}

Table 2. The analysis table of measurement results.

\begin{tabular}{|c|c|c|c|c|}
\hline Experiment & Spot Size (pixel) & Estimated Distance(cm) & Actual Distance(cm) & Relative Error(\%) \\
\hline 1 & 122.324 & 118.312 & 119 & 0.578 \\
\hline 3 & 198.205 & 220.023 & 221 & 0.442 \\
\hline 5 & 301.212 & 311.144 & 313 & 0.593 \\
\hline 6 & 350.265 & 365.232 & 366 & 0.210 \\
\hline 7 & 390.113 & 397.264 & 399 & 0.435 \\
\hline 8 & 408.229 & 409.231 & 411 & 0.430 \\
\hline 11 & 594.624 & 508.233 & 510 & 0.346 \\
\hline 12 & 632.205 & 521.106 & 523 & 0.362 \\
\hline 13 & 682.506 & 580.229 & 582 & 0.304 \\
\hline 14 & 741.026 & 640.118 & 642 & 0.293 \\
\hline 15 & 789.205 & 698.203 & 700 & 0.257 \\
\hline
\end{tabular}


Meanwhile, this method was available to the displacement measurement of adaptive wing to measure the changing displacement effectively, improve the speed measurement and make results in the range of requirements.

\section{CONCLUSION}

The method proposed in this paper is simple to operate and easy to calculate, and could be used for non-contract real-time measurement in range finding. Now it is applied in displacement measurement of adaptive wing successfully.

In the measurement system, the errors come from two aspects mainly: first the influence on the quality of laser image because of the changing environment and roughness on the surface of object, and the inaccuracy of spot extraction because of the optical characteristics of laser beam, such as inherent drift and so on. Second the problem of camera, such as the installation, length of focus, the alignment and so on. If these factors could be removed, the accuracy of experiment would be better.

\section{CONFLICT OF INTEREST}

The author confirms that this article content has no conflict of interest.

\section{REFERENCES}

[1] H. Xianli, and X. Guiyang, "Study on digital image median filtering technique", Electron. Design Eng., pp. 191-193, June 2014.

[2] W. Jian and C. Hongbin, "A Three-dimensional Auto-focusing System Based on Image Processing". Opto-Electron. Eng., pp. 4651, Feb 2014.

[3] C. Yemei, "Image Collection System Design Based on Embedded System" J. ChangSha Aeronaut. Vocat. Technical College, pp. 6062, March 2011.

[4] Z. Jigui, and Z. Jian, "Photogrammetric precision error compensation method for image processing”, pp. 122-129, 2012.

[5] Z. Ying, "Application of computer imagery processing in holography", Manufact. Automat., pp. 90-92, Sep 2011.

[6] W. Chenghe, and B. Chunhua, Z. Ting, "Research on the Algorithm for Crack Detection in Slab Track Based on Digital Image Processing”, Electro-Optic Technol. Appl., pp. 49-51, Feb 2014.

[7] Y. Jing, "Mathematical Model Based on Super Resolution Reconstruction of Medical Image Processing Method", Comput. Simulat., pp. 243-245, May 2014.

[8] L. Rui, and W. Xiaochun. "Automatic Identify of Linear Tracks Based on Digital Image Processing", Video Eng., pp. 167-169, March 2014.

[9] Z. Che, and X. Xiping, "A study on a measurement method based on image processing", Electronic Test, pp. 110-112, July 2014.

[10] W. Zhenyan, "Cracks Automatic Identification and Evaluation System Based on Digital Image Processing", Comput. Eng. Software, pp. 149-151, Jan 2014.

[11] W. Wei, and D. Fajie, "On-line detecting system of gaps in screen based on image progressing technology", Appl Electron. Technique, pp. 120-122, Apr 2014.

\section{ACKNOWLEDGEMENTS}

Declared none.

Received: September 16, 2014

Revised: December 23, 2014

Accepted: December 31, 2014

(C) Xie and Ma; Licensee Bentham Open.

This is an open access article licensed under the terms of the Creative Commons Attribution Non-Commercial License (http://creativecommons.org/licenses/ by-nc/4.0/) which permits unrestricted, non-commercial use, distribution and reproduction in any medium, provided the work is properly cited. 